\title{
Osteonecrosis in Primary Antiphospholipid Syndrome is Associated with Previous Glucocorticoid Use and Thrombocytopenia
}

\author{
Jozélio Freire de Carvalho (D) - Roberto Paulo Correia de Araujo • \\ Thelma Larocca Skare
}

Received: March 22, 2021 / Accepted: June 10, 2021 / Published online: July 6, 2021

(C) The Author(s) 2021

\section{ABSTRACT}

Introduction: The aim of this study was to investigate the frequency of avascular necrosis $(\mathrm{AVN})$ and its associations with clinical and laboratory parameters in patients with primary antiphospholipid antibody syndrome (pAPS).

Methods: This was a cross-sectional study of 66 patients with pAPS who fulfilled the Sidney criteria for APS. Epidemiological and clinical data, and data on antiphospholipid antibodies were collected. Diagnosis of AVN was based on studies of magnetic resonance (MRI) images of affected bones.

Results: AVN was found in three of the 66 (4.5\%) enrolled patients. Comparison of patients with pAPS with and without AVN showed that demographic data, comorbidities, and antiphospholipid antibodies profile were similar in both groups. Compared to patients without AVN, those with AVN had thrombocytopenia more frequently (66.7 vs. $7.9 \%)$ and used more glucocorticoids (100 vs. 35\%).

J. Freire de Carvalho $(\varangle) \cdot$ R. P. Correia de Araujo Institute for Health Sciences, Federal University of Bahia, Rua das Violetas, 42, ap. 502, Pituba, Salvador, BA, Brazil

e-mail: jotafc@gmail.com

T. L. Skare

Unit of Rheumatology, Hospital Evangélico

Mackenzie, Curitiba, PR, Brazil
Conclusion: AVN is rare in patients with pAPS and may be associated with thrombocytopenia and previous glucocorticoid use.

Keywords: Antiphospholipid syndrome; Antiphospholipid antibodies; Osteonecrosis; Thrombosis

\section{Key Summary Points}

Osteonecrosis is described in patients with antiphospholipid antibody syndrome

In this study, patients with osteonecrosis had thrombocytopenia more frequently and used more glucocorticoids than those without osteonecrosis

\section{DIGITAL FEATURES}

This article is published with digital features, including a summary slide, to facilitate understanding of the article. To view digital features for this article go to https://doi.org/10.6084/ m9.figshare.14749443. 


\section{INTRODUCTION}

Antiphospholipid antibody syndrome (APS) is an autoimmune acquired thrombophilic disorder that is characterized by vascular thrombosis and/or obstetric events associated or not with thrombocytopenia, occurring in patients with persistent moderate to high levels of antiphospholipid antibodies [1]. Vascular thrombosis is the hallmark of this disease, but other manifestations, such as movement disorders, cognitive impairments, convulsions, cardiac lesions, and multiple sclerosis-like symptoms, not linked to thrombosis may also be found [2]. Avascular necrosis (AVN) has also been associated to APS, although the nature of this association is not completely clear [3]. AVN is a skeletal disease of unknown pathophysiology characterized by the death of bone marrow cells and trabecular bone. It results in the collapse of the architectural bone structure, leading to joint pain, bone destruction, and function loss. Circulatory impairment of the affected bone has been assumed to be the common denominator for all cases of AVN [4]. In APS, thrombosis of bone vessels is considered a possible mechanism, although patients with APS may have a number of other risk factors, such as glucocorticoid use, dyslipidemia, trauma, among others, making comprehension of the underlying process more difficult [4].

A cross-sectional study with magnetic resonance imaging (MRI) of the femoral head in 30 asymptomatic patients with primary APS (pAPS) showed that $20 \%$ of the patients had signs suggesting the presence of AVN and that these signs were more common in those patients with livedo reticularis [3]. Cervera et al. [5] revealed a prevalence of $0.9 \% \mathrm{AVN}$ in their study population of 538 patients with pAPS. In contrast, Mok et al. [6], who studied 265 patients with systemic lupus, found that the presence of antiphospholipid antibodies was not linked to the occurrence of AVN in the $4 \%$ of the sample group affected by this complication. Likewise, Vianna et al. [7] did not note any AVN cases among their 58 patients with pAPS observed during 5 years.
We report here the results of our study on the frequency of AVN in a sample of 66 patients with pAPS. The aim of this study was to determine the frequency of AVN in a group of Brazilian patients with pAPS and identify possible associations with the epidemiological, clinical, and serological profile.

\section{METHODS}

All patients included in this study signed an informed consent form and were recruited from the first author's private practice. The authors declare that the study conformed with the Helsinki Declaration of 1964 and subsequent revisions. The ethical committee of Federal University of Bahia approved this study.

Inclusion criteria were age $>18$ years and fulfillment of the Sidney classification criteria for APS [8].

Data collection included: (1) epidemiological and anthropometrical data [age, sex, ethnic background, weight and height for body mass index (BMI) calculation, exercise history (yes/ no), and smoking history]; (2) clinical data (arterial and venous thrombosis, obstetric history, presence of Sneddon syndrome, livedo reticularis, thrombocytopenia, and comorbidities).

All arterial and venous thrombotic events were documented by imaging methods (e.g. Doppler ultrasound, ventilation/perfusion pulmonary scan, tomography or nuclear magnetic resonance, arteriography, computed tomography pulmonary angiogram, MRI angiography). Thrombocytopenia was defined as platelet count $<100,000 / \mathrm{mm}^{3}$ on at least two occasions. Sneddon's syndrome was defined as the presence of stroke and livedo racemosa [9].

The comorbidities considered were: arterial hypertension (blood pressure $>140 \times 90 \mathrm{mmHg}$ or use of anti-hypertensive drugs); dyslipidemia (total cholesterol $>200 \mathrm{mg} / \mathrm{dL}$, triglycerides > $150 \mathrm{mg} / \mathrm{dL}$, low-density lipoprotein $>160 \mathrm{mg} /$ $\mathrm{dL}$ or high-density lipoprotein-cholesterol < $40 \mathrm{mg} / \mathrm{dL}$ ). AVN was diagnosed on the basis of clinical findings and the diagnosis confirmed by MRI imaging.

Data on treatments used were also collected. 
Laboratory data included the presence and titer of anticardiolipin (aCL) immunoglobulin (Ig) G and IgM and lupus anticoagulant (LA). The patient was considered to be positive for aCL when aCL titers were $\geq 40$ IgG phospholipid units (GPL) or IgM phospholipid units (MPL) on at least two occasions, 12 weeks apart. aCL was measured using commercial kits from Phadia, ThermoFisher Scientific (Waltham, MA, USA). LA was measured according to the international guidelines using activated partial thromboplastin time (aPTT; Diagnostica Stago, Asnières-sur-Seine, France), and diluted Russel's viper venom time (dRVVT; Trinity Biotech, Wiclow, Ireland) [10]. Tests for the presence of lupus-related antibodies (anti-dsDNA, antichromatin, anti-Ro/SS-A, anti-La/SS-B, anti-Sm) were performed in all cases and all test results were negative.

MRI was performed in patients with clinical suspicion of AVN.

The statistical analysis included frequency (\%) of nominal variables and median (minimum-maximum) numerical variables. Mann-Whitney tests were used to compare numerical data, and the Fisher test was used to compare frequencies. The analysis was done using JASP software version 0.12.2. $p$ values < 0.05 were considered to be significant.

\section{RESULTS AND DISCUSSION}

Of the 66 patients with pAPS who fulfilled the Sidney criteria for pAPS enrolled in this study, three had AVN. Comparison of the anthropometrical and epidemiological data revealed no statistically significant differences between those with and without AVN (Table 1). The median follow-up of all patients was 7 (range 2-10) years.

The frequency of AVN among the 66 patients $(4.5 \%)$ in our study is lower than that reported by Tektonidou et al. [3], who found that of the 30 patients with pAPS in their study population, $20 \%$ had AVN. These authors investigated asymptomatic cases using femur MRI [3], while we limited enrolment to patients with clinical symptoms of pAPS in order to replicate as closely as possible the real-world scenario.
Asherson et al. [11] studied a cohort of 70 patients with pAPS and found that 3\% had $\mathrm{AVN}$, which is a frequency similar to that in our study.

Clinical data for the two patient groups were compared and the results are given in Table 2. The values show that patients with AVN had more thrombocytopenia than those without it. All patients had unilateral osteonecrosis of the hips.

Comparison of drug treatment in the Table 3 shows that in individuals with AVN, the previous use of glucocorticoid was more common in those patients than those without AVN (100\% vs. $34.9 \%, p=0.038)$. Patients used glucocorticoid due to thrombocytopenia $(n=7)$, in a median dose of $30 \mathrm{mg} /$ day that was tapered off in 6 months; and other several cases $(n=18)$ used this drug at the beginning of the diagnosis, before primary APS diagnosis was confirmed. This drug was rapidly tapered off. The mean time of glucocorticoid (GC) use of these subjects was about 4 months.

AVN has been linked to fat embolism, vascular thrombosis, coagulopathy, and fatigue fractures [12]. It is also well known that glucocorticoids are associated with AVN, although the underlying pathogenesis is not well studied [13]. One possible mechanism are alterations in lipid levels resulting in fat microemboli in the arteries supplying bone [14]. Another proposed mechanism is increases in the number and the size of bone marrow adipocytes, resulting in increased intraosseous pressure that blocks venous outflow [15]. A third theory is that glucocorticoids could induce changes in venous endothelial cells, thereby increasing stasis and intraosseous pressure and predisposing to necrosis [16].

An essential driver of the appearance of AVN, however, appears to be the apoptosis of osteoblasts. Glucocorticoid-induced osteocyte apoptosis disrupts the osteocyte-lacunar-canalicular system's mechanosensory function, resulting in the initiation of a sequence of actions that lead to collapse of the femoral head [12, 17]. Abundant apoptotic osteocytes have been found in the tissue of femoral heads removed at hip surgery, as a result of glucocorticoid-induced osteonecrosis [18]. 
Table 1 Comparison of clinical and epidemiological data of patients with primary antiphospholipid antibody syndrome with and without avascular necrosis

\begin{tabular}{llll}
\hline $\begin{array}{l}\text { Clinical and epidemiological } \\
\text { parameters }\end{array}$ & $\begin{array}{l}\text { pAPS patients with AVN } \\
(\boldsymbol{n}=\mathbf{3})\end{array}$ & $\begin{array}{l}\text { pAPS patients without AVN } \\
(\boldsymbol{n}=\mathbf{6 3})\end{array}$ & $\boldsymbol{p}$ value \\
\hline $\begin{array}{l}\text { Median age, years } \\
\quad(\text { minimum-maximum) }\end{array}$ & $43(34-44)$ & $39(22-66)$ & 0.75 \\
Female sex, $n$ (\%) & $n(67)$ & $n-(84)$ & 0.18 \\
$\begin{array}{l}\text { Median BMI, kg/m } \\
\quad(\text { minimum-maximum) }\end{array}$ & $29(24.3-31)$ & $27.6(17.7-50.5)$ & 0.56 \\
$\begin{array}{l}\text { Physically active, yes/no, } n(\%) \\
\text { Current smokers, } n(\%)\end{array}$ & $1(33.3)$ & $17(26.9)$ & 1.00 \\
Ex-smokers, $n(\%)$ & 0 & $7(11.1)$ & 1.00 \\
\hline
\end{tabular}

$A V N$ Avascular necrosis, $B M I$ body mass index, $p A P S$ primary antiphospholipid antibody syndrome

Table 2 Comparison of clinical data of patients with pAPS with and without AVN

\begin{tabular}{llll}
\hline Clinical data, $\boldsymbol{n}(\%)$ & pAPS patients with AVN $(\boldsymbol{n}=\mathbf{3})$ & pAPS patients without AVN $(\boldsymbol{n}=\mathbf{6 3})$ & $\boldsymbol{p}$ value $^{\mathbf{a}}$ \\
\hline Arterial events & $2(66.7)$ & $36(57.1)$ & 1.00 \\
Venous events & $1(33.3)$ & $30(47.6)$ & 1.00 \\
Obstetrical events & $1(33.3)$ & $20(31.7)$ & 0.47 \\
Strokes & $2(66.7)$ & $23(36.5)$ & 0.64 \\
Sneddon's syndrome & 0 & $13(20.6)$ & 0.76 \\
Livedo reticularis & 0 & $18(28.6)$ & 0.54 \\
Limb ischemi & 0 & $10(15.9)$ & 0.98 \\
Miocardial infarction & 0 & $4(6.3)$ & 1.00 \\
Angina & 0 & $3(4.8)$ & 1.00 \\
Profound venous thrombosis & $1(33.3)$ & $40(63.5)$ & 0.64 \\
Pulmonary embolism & 0 & $15(23.8)$ & 0.72 \\
Thrombocytopenia & $2(66.7)$ & $5(7.9)$ & $0.02^{*}$ \\
Arterial hypertension & $1(33.3)$ & $24(38.1)$ & 1.00 \\
Dyslipidemia & $2(33.3)$ & $18(28.6)$ & 0.29 \\
\hline
\end{tabular}

Values in table are presented as the number $(n)$ with the percentage in parentheses

*Significant difference between patient groups at $p<0.05$

${ }^{a} p$ values were calculated using the Fisher test

Some of the pathobiological mechanisms leading to thrombosis in APS that may play an essential role in the pathophysiological mechanisms of AVN include inhibition of the protein $\mathrm{C}$ anticoagulant pathway, endothelial injury and/or pathological activation, 
Table 3 Comparison of treatments for patients with pAPS with and without AVN

\begin{tabular}{llll}
\hline Comparison of treatments & pAPS patients with AVN $(\boldsymbol{n}=\mathbf{3})$ & pAPS patients without AVN $(\boldsymbol{n}=\mathbf{6 3})$ & $\boldsymbol{p}$ value $^{\mathbf{a}}$ \\
\hline Glucocorticoid (current use) & $1(33.3)$ & $5(7.9)$ & 0.601 \\
Glucocorticoid (previous use) & $3(100)$ & $22(34.9)$ & $0.038^{*}$ \\
Warfarin & $3(100)$ & $49(77.8)$ & 0.87 \\
Antimalarials & $1(33.3)$ & $12(19.0)$ & 1.00 \\
Statin & $2(66.7)$ & $15(23.8)$ & 0.32 \\
Acetylsalicylic acid (current use) & $1(33.3)$ & $36(57.1)$ & 0.56 \\
\hline
\end{tabular}

Values in table are presented as the number $(n)$ with the percentage in parentheses

*Significant difference between patient groups at $p<0.05$

${ }^{a} p$ values were calculated using the Fisher test

alterations in the tissue factor pathway, molecular mimicry, platelet activation, increased fibrin polymerization rate, impaired fibrinolytic activity, inhibition of the formation of antithrombin III-thrombin complexes, among others. This topic is extensively reviewed by Öztürk et al. [19].

The prescription of glucocorticoid in primary APS is controversial. In our patient sample, it was used to treat thrombocytopenia and renal microangiopathy, and may have contributed to the appearance of AVN.

Interestingly, one of the patients with AVN in our study tested positive for the Factor $\mathrm{V}$ Leiden mutation. Thrombophilias are also considered to be risk factors for AVN. An Indian study demonstrated the presence of protein $\mathrm{C}$ and $S$ deficiencies in patients with AVN compared to the controls, but there were no differences between the groups regarding the presence of the Factor V Leiden mutation [20]. In a study carried out in a Spanish population of 29 patients with multifocal AVN, 26 patients had associated prothrombotic abnormalities, but only one had a Factor V Leiden mutation [21]. The authors considered this prevalence to be similar to that in the general population [21]. No patient in our study population had a previous history of decompression, sickle cell anemia, trauma, human immunodeficiency virus, and/or heavy alcoholism.

Another significant association observed in our study was that of AVN and thrombocytopenia. Thrombocytopenia is the most common non-criteria hematologic manifestation of APS, with a frequency fluctuating from 20 to $50 \%$. [21]. It is considered to be secondary in cases with an increased platelet activation and destruction or decreased platelet production, or even increased platelet pooling [21]. The activation of platelets and endothelial cells produces a prothrombotic state responsible for the AVN [22]. However, it is also possible that this association reflects the underlying use of glucocorticoids for this type of manifestation.

The presence of positive aCL IgG (67 vs. 83\%; $p=0.15) \operatorname{IgM}(100$ vs. $83 \% ; p=0.14)$ and LA (67 vs. $73 \% ; p=0.19$ ) was similar in patients with and without AVN. Two of the three patients with AVN had triple positivity for antiphospholipid antibodies (IgG, IgM, and LA), and one of the three had double positivity (IgG and IgM $\mathrm{aCL})$.

This study has a number of limitations. First, it is a cross-sectional design and as such it does not determine cause-effect relationships. Second, the number of patients was limited to those with AVN, which may induce bias in interpreting comparisons. Third, all patients with AVN also had previous glucocorticoid use; thus, the findings may also be due to the effects of glucocorticoid treatment. However, even in glucocorticoid users, positivity for antiphospholipid antibodies seems to increase the risk for AVN [23]. Lastly, the MRI examination was 
performed only in symptomatic patients with a clinical suspicion of AVN.

\section{CONCLUSIONS}

The results show that only a small proportion of the patients with pAPS included in the study had AVN and that this complication was more common in those patients using/having used glucocorticoids and presenting a low platelet count. These findings suggest that the presence of AVN is linked to previous glucocorticoid use and thrombocytopenia. Therefore, we suggest that patients with these characteristics should be closely observed for AVN. However, since our study involved a relatively small number of patients with AVN, future studies with a higher number of patients are needed to confirm the associations found herein.

\section{ACKNOWLEDGEMENTS}

We thank the participants of the study.

Funding. No funding or sponsorship was received for this study or publication of this article.

Authorship. All named authors meet the International Committee of Medical Journal Editors (ICMJE) criteria for authorship for this article, take responsibility for the integrity of the work as a whole, and have given their approval for this version to be published.

Authors' Contributions. JFC: Conception, data analysis, statistical analysis, writing, submission. RPCA: data analysis, revision. TLS: Data analysis, writing, revision.

Disclosures. Jozélio Freire Carvalho, Roberto Paulo Correia de Araujo, and Thelma Larocca Skare have nothing to disclose.

Compliance with Ethics Guidelines. All patients included in this study signed an informed consent form and were recruited from the first author's private practice. The authors declare that the study conformed with the Helsinki Declaration of 1964 and subsequent revisions. The ethical committee of Federal University of Bahia approved this study.

Data Availability. The datasets generated during and/or analyzed during the current study are available from the corresponding author on reasonable request.

Open Access. This article is licensed under a Creative Commons Attribution-NonCommercial 4.0 International License, which permits any non-commercial use, sharing, adaptation, distribution and reproduction in any medium or format, as long as you give appropriate credit to the original author(s) and the source, provide a link to the Creative Commons licence, and indicate if changes were made. The images or other third party material in this article are included in the article's Creative Commons licence, unless indicated otherwise in a credit line to the material. If material is not included in the article's Creative Commons licence and your intended use is not permitted by statutory regulation or exceeds the permitted use, you will need to obtain permission directly from the copyright holder. To view a copy of this licence, visit http://creativecommons.org/licenses/by$\mathrm{nc} / 4.0 /$.

\section{REFERENCES}

1. Sammaritano LR. Antiphospholipid syndrome. Best Pract Res Clin Rheumatol. 2020;34(1):101463.

2. Xourgia E, Tektonidou MG. Management of noncriteria manifestations in antiphospholipid syndrome. Curr Rheumatol Rep. 2020;22(9):51.

3. Tektonidou MG, Malagari K, Vlachoyiannopoulos PG, Kelekis DA, Moutsopoulos HM. Asymptomatic avascular necrosis in patients with primary antiphospholipid syndrome in the absence of corticosteroid use: a prospective study by magnetic resonance imaging. Arthritis Rheum. 2003;48(3): 732-6.

4. Rueda JC, Duque MA, Mantilla RD, Iglesias-Gamarra A. Osteonecrosis and antiphospholipid syndrome. J Clin Rheumatol. 2009;15(3):130-2. 
5. Cervera R, Piette JC, Font J, Khamashta MA, Shoenfeld Y, Camps MT, et al. Antiphospholipid syndrome: clinical and immunologic manifestations and patterns of disease expression in a cohort of 1,000 patients. Arthritis Rheum. 2002;46: 1019-27.

6. Mok MY, Farewell VT, Isenberg DA. Risk factors for avascular necrosis of bone in patients with systemic lupus erythematosus: Is there a role for antiphospholipid antibodies? Ann Rheum Dis. 2000;59(6): 462-7.

7. Weinstein RS. Glucocorticoid-induced osteonecrosis. Endocrine. 2012;41(2):183-90.

8. Miyakis S, Lockshin MD, Atsumi T, Branch DW, Brey RL, Cervera R, et al. International consensus statement on an update of the classification criteria for definite antiphospholipid syndrome (APS). J Thromb Haemost. 2006;4(2):295-306.

9. Samanta D, Cobb S, Arya K. Sneddon syndrome: a comprehensive overview. J Stroke Cerebrovasc Dis. 2019;28(8):2098-108.

10. Wisloff F, Jacobsen EM, Liestol S. Laboratory diagnosis of the antiphospholipid syndrome. Thromb Res. 2002;108:263-71.

11. Asherson RA, Khamashta MA, Ordi-Ros J, Derksen $\mathrm{RH}$, Machin SJ, Barquinero J, et al. The "'primary"' antiphospholipid syndrome: major clinical and serological features. Medicine. 1989;68:366-74.

12. Weinstein RS. Clinical Practice: glucocorticoid-induced bone disease. N Engl J Med. 2011;365:62-70.

13. Wang A, Ren M, Wang J. The pathogenesis of steroid-induced osteonecrosis of the femoral head: a systematic review of the literature. Gene. 2018;671: 103.

14. Jones JP Jr. Fat embolism and osteonecrosis. Orthop Clin North Am. 1985;16:595-633.
15. Solomon L. Idiopathic necrosis of the femoral head: pathogenesis and treatment. Can J Surg. 1981;24: 573-8.

16. Nishimura T, Matsumoto T, Nishino M, Tomita K. Histopathologic study of veins in steroid-treated rabbits. Clin Orthop Relat Res. 1997;334:37-42.

17. Colwell CW Jr, Robinson CA, Stevenson DD, Vint VC, Morris BA. Osteonecrosis of the femoral head in patients with inflammatory arthritis or asthma receiving corticosteroid therapy. Orthopedics. 1996;19:941-6.

18. Weinstein RS, Nicholas RW, Manolagas SC. Apoptosis of osteocytes in glucocorticoid-induced osteonecrosis of the hip. J Clin Endocrinol Metab. 2000;85:2907-12.

19. Oztürk MA, Haznedaroğlu IC, Turgut M, Göker H. Current debates in antiphospholipid syndrome: the acquired antibody-mediated thrombophilia. Clin Appl Thromb Hemost. 2004;10(2):89-126.

20. Rathod TN, Tayade MB, Shetty SD, Jadhav P, Sathe $\mathrm{AH}$, Mohanty SS. Association of thrombophilic factors in pathogenesis of osteonecrosis of femoral head in Indian population. Indian $\mathrm{J}$ Orthop. 2020;54(Suppl 1):33-8.

21. Gómez-Puerta JA, Peris P, Reverter JC, Espinosa G, Martinez-Ferrer A, Monegal A, et al. High prevalence of prothrombotic abnormalities in multifocal osteonecrosis: description of a series and review of the literature. Medicine (Baltimore). 2013;92(6): 295-304.

22. Artim-Esen B, Diz-Küçükkaya R, İnanç M. The significance and management of thrombocytopenia in antiphospholipid syndrome. Curr Rheumatol Rep. 2015;17(3):14.

23. Galindo M, Mateo I, Pablos JL. Multiple avascular necrosis of bone and polyarticular septic arthritis in patients with systemic lupus erythematosus. Rheumatol Int. 2005;25:72-6. 